\title{
Especialización de los sistemas productivos lecheros en México: la difusión del modelo tecnológico Holstein
}

\section{Specialization of dairy production systems in Mexico: Diffusion of Holstein technological model}

\author{
Joaquín Hiutzilihuitl Camacho Veraa ${ }^{a}$ Fernando Cervantes Escoto ${ }^{a^{*}}$, María Isabel Palacios Rangel ${ }^{a}$, \\ Alfredo Cesín Vargas ${ }^{b}$, Jorge Ocampo Ledesma ${ }^{a}$
}

\begin{abstract}
RESUMEN
En México, el sector de producción de leche de bovino se caracteriza por su concentración espacial en determinadas regiones. En el país se tienen tres sistemas de producción de leche diferenciados geográficamente: intensivo, familiar y extensivo de doble propósito. Este trabajo plantea que durante la última década se ha modificado la configuración espacial de la actividad lechera, lo que ha generado un avance de la especialización regional atribuible al avance del modelo tecnológico Holstein. Para analizarlo, se utilizaron dos indicadores de economía regional con el fin de detectar cambios en los patrones y en la estructura productiva. Se utilizó el cociente de localización (CL) y el análisis "Shift and Share", tomando el valor de la producción agrícola y pecuaria como la variable de análisis. Los resultados muestran que entre 2002 y 2013 se dio una reconfiguración de la especialización regional de producción de leche en el país. Se incrementaron las regiones especializadas, lo que implica una mayor preponderancia con respecto a otras actividades agrícolas y pecuarias. Regiones como La laguna, (DDR Laguna Durango y Laguna Coahuila) han mantenido su alta especialización lechera; al mismo tiempo, entidades como Chihuahua y Durango se han consolidado como regiones de alta especialización productiva. Incluso sistemas de doble propósito, como los del norte del estado de Veracruz y Chiapas, han modificado su estructura productiva hacia la producción de leche de bovino. Se concluye que durante el periodo de análisis hubo un fuerte avance en la especialización lechera en el país.
\end{abstract}

PALABRAS CLAVE: Cociente de localización, Especialización regional, modelo Holstein, Shift and Share.

\section{ABSTRACT}

In Mexico, the production of bovine milk is characterized by its spatial concentration in certain regions. The country has three production systems geographically differentiated: intensive, family farm and extensive/dual purpose. This work suggests that over the last decade has changed the spatial configuration of dairy farming, which has generated an advance of regional specialization attributable to advance of the Holstein model. To detect patterns and changes in the production structure two indicators of regional economy were used. The value of production (crop and livestock) and an analysis Shift and Share were used to calculate the location quotient. The results show that between 2002 and 2013 a reconfiguration of the regional specialization of milk production in the country was given. Specialized dairy regions increased, implying a greater preponderance over other agricultural and livestock activities. Regions such as La Laguna, (DDR Laguna Durango y Laguna Coahuila) have maintained their high milk specialization. Meanwhile, entities such as Chihuahua and Durango were consolidated as productive regions of high specialization. Even dual-purpose systems, such as the northern state of Veracruz and Chiapas, have modified their production structure towards the production of bovine milk. It concludes that during the analysis period, there was a strong increase in milk production specialization in the country.

KEY WORDS: Location quotient, Regional specialization, Holstein model, Shift and Share.

Recibido el 24 de mayo de 2016. Aceptado el 13 de septiembre de 2016.

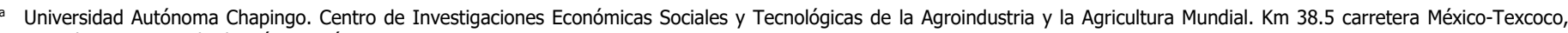
56230, Chapingo, Estado de México. México.

b Universidad Nacional Autónoma de México. Unidad Académica de Estudios Regionales de la Coordinación de Humanidades. Jiquilpan, Michoacán. México.

*Autor de correspondencia: tartalian04@gmail.com. 


\section{INTRODUCCIÓN}

La expansión global de la industria alimentaria ocurrida desde mediados de siglo XX forma parte de un proceso de consolidación de una "modernidad alimentaria", en la que los laboratorios y plantas agroindustriales se han apropiado de la producción de alimentos ${ }^{(1)}$. La globalización ha generado una dependencia creciente de la agricultura hacia la agroindustria, creando lazos que llegan incluso a niveles de subordinación. Como resultado, se han dado procesos de regionalización comercial y de producción, que han consolidado la posición de las empresas de los países industrializados ${ }^{(2)}$. En América del Norte, Estados Unidos ha favorecido una integración comercial con México y Canadá, por medio de la firma de acuerdos comerciales como el Tratado de Libre Comercio con América del Norte (TLCAN), a fin de distribuirse los mercados de bienes y servicios ${ }^{(3)}$.

Hasta mediados del siglo XX, en las distintas regiones del mundo, cada sistema lechero se desarrollaba de manera independiente. No obstante, el desarrollo y la adopción de una serie de tecnologías permitieron un importante aumento de la producción, además de la posibilidad de comercializar a grandes distancias ${ }^{(3)}$. Al paradigma tecnológico que incorpora los avances científicos para hacer intensiva la producción, se le ha denominado modelo Holstein. Este modelo nace a principios del siglo $\mathrm{XX}$, impulsado por diversas investigaciones sobre reproducción asistida, sin embargo, es hasta 1930 cuando se desarrolla de manera plena en occidente. A la par, fueron incorporados otros avances científicos y tecnológicos relacionados con la producción y uso de antibióticos y con la conservación de alimentos ${ }^{(4,5)}$. No fue sino hasta principios de 1980 cuando, de la mano del control de la agroindustria sobre la producción primaria, el modelo Holstein se consolidó como el paradigma tecnológico predominante en la producción de leche.

En México, la producción de leche se ha concentrado en cuencas especializadas, desde donde se distribuye a los grandes centros urbanos de consumo. El sector se caracteriza por su concentración espacial y su relevancia económica ${ }^{(6)}$. Se presentan tres sistemas diferenciados geográficamente: a) intensivo, que comprende la Comarca Lagunera, Durango y Coahuila, recientemente Querétaro; b) familiar, todo el altiplano central y, c) extensivo de doble propósito, presente en las regiones tropicales ${ }^{(7)}$. La organización de la producción de leche en el ámbito nacional es un reflejo fiel de lo que ocurre en el mundo: procesos de concentración de la producción, y control de la agroindustria.

El modelo Holstein altamente tecnificado se ha vinculado de forma muy estrecha con la gran industria lechera nacional y transnacional ${ }^{(8)}$. La hipótesis que se sostuvo en este trabajo es que durante la última década se han registrado avances significativos del modelo, que han influido no solo en la estructura de la producción de las distintas regiones, sino también, en la ordenación agraria local, con lo que se ha modificado su configuración nacional y se ha generado una importante especialización espacial.

Se realizó un análisis del comportamiento de la actividad lechera en el país con el fin de detectar los patrones de especialización espacial en la producción y procesos de cambio. Para tal objetivo se asumieron los conceptos y categorías de la modernidad alimentaria ${ }^{(1)}$ como guía para discutir los resultados. Se utilizaron indicadores de Economía Regional que valoran la distribución de las actividades económicas y su estructura relativa (cociente de localización), así como su comportamiento en el tiempo mediante el análisis "Shift and Share"(9).

\section{MATERIAL Y MÉTODOS}

En este estudio se tomaron a los distritos de desarrollo rural (DDR) como las unidades geográficas fundamentales para analizar las disparidades regionales en cuanto a especialización lechera. Para tal fin, se determinó el cociente de localización (CL) y el análisis de cambio y participación utilizando el valor de la producción para su cálculo. Se construyó una base de datos con información obtenida del Servicio de Información Agroalimentaria y Pesquera (SIAP) en relación al valor de la producción del sector agropecuario para cada Distrito de Desarrollo Rural, para 2002 y 2013. 
Los subsectores pecuarios se consideraron por separado, y todo el subsector agrícola como un solo bloque, considerando que el foco del análisis fue el subsector de producción de leche de bovino. La información se organizó a manera de una matriz de doble entrada sector-región (SECREG), colocando los distintos sectores agropecuarios en las columnas y las regiones en las filas. Una vez construida la matriz SECREG, se procedió a realizar el cálculo del CL para 2002 y 2013, y el análisis de cambio y participación para los incrementos en el sector lechero en el mismo periodo ${ }^{(10)}$ haciendo uso de la siguiente expresión:

$$
\mathrm{CL}_{\mathrm{i}}=\left(\mathrm{X}_{\mathrm{ij}} / \Sigma_{\mathrm{i}} \mathrm{X}_{\mathrm{ij}}\right) /\left(\Sigma_{\mathrm{j}} \mathrm{X}_{\mathrm{ij}} / \Sigma_{\mathrm{i}} \Sigma_{\mathrm{j}} \mathrm{X}_{\mathrm{ij}}\right)
$$

En la que $\mathrm{CL}_{i}$ representa al cociente de localización, $\mathrm{X}$ es la variable de análisis, i corresponde al sector y $j$ corresponde a la región. De acuerdo con este cociente, se puede afirmar que existe especialización relativa del sector $i$ en la región $j$ cuando su valor es mayor que $1^{(9,10)}$. El coeficiente se interpreta como una medida de concentración geográfica, que ubica la primacía de cada sector dentro de un conglomerado de regiones. Entre más se acerque a cero habrá un menor grado de concentración del sector en la región y viceversa.

De la aplicación de esta metodología se obtuvo una matriz con los cocientes de localización de cada actividad para cada región considerada (en este caso, los distritos de desarrollo con actividad lechera). Se tomó únicamente la columna que correspondía al sector región y se asoció con las capas vectoriales que correspondían a cada DDR. De esa asociación se obtuvieron los mapas que mostraron los distritos especializados ( $C L>1)$ subdivididos en especializados y muy especializados según los resultados obtenidos del análisis de conglomeración. Se realizó el análisis de conglomerados con el fin de determinar tres niveles de especialización. Se utilizó el agrupamiento por conglomerados jerárquicos por medio del método de "vecinos más lejanos"11), dado que éste permite evitar inconsistencias e indefiniciones en la formación de grupos. Todos los distritos de desarrollo rural con un $\mathrm{CL}$ menor a uno se consideraron como no especializados, y se les identificó con color blanco.
Para el cálculo del indicador de cambio y participación (Shift and Share), se utilizaron las siguientes identidades ${ }^{(10)}$ :
1) $X^{\prime}{ }_{i j}-X_{i j}=\Delta X_{i j}$ $=X_{i j} r+X_{i j}\left(r_{i}-r\right)+X_{i j}\left(r_{i j}-r\right)$
2) $r=\frac{\sum_{i=1}^{S} \sum_{j=1}^{R}\left(X^{\prime}{ }_{i j}-X_{i j}\right)}{\sum_{i=1}^{S} \sum_{j=1}^{R} X_{i j}}$
3) $r_{i}=\frac{\sum_{j=1}^{R}\left(X_{i j}^{\prime}-X_{i j}\right)}{\sum_{j=1}^{R} X_{i j}}$
4) $r_{i j}=\frac{X_{i j}^{\prime}-X_{i j}}{X_{i j}}$

En la primera ecuación el término $\left(\mathrm{X}_{\mathrm{ij}}{ }^{\mathrm{r}}\right)$ corresponde al efecto total; el segundo $\left(X_{i j}\left(r_{i}-r\right)\right)$ recae en el sectorial o estructural, y el tercero $\left(X_{i j}\left(r_{i i-}\right.\right.$ r)) concierne al regional o competitivo. El análisis de cambio y participación evalúa el diferencial del crecimiento de los sectores analizados en las regiones estudiadas. Éste es uno de los métodos de análisis dinámico más usado, en virtud de sus posibilidades analíticas y lo elemental de la información necesaria para construirlo ${ }^{(12)}$. Fue planteado en la década de los sesenta, con lo cual se trató de responder preguntas tales como, cuáles regiones muestran mayor crecimiento, y si este último se puede atribuir a: i) un efecto global (suma de regiones); ii) un efecto sectorial (estructural); iii) un efecto regional (competitivo) $)^{(13)} \mathrm{o}$, iv) es la suma, cuantitativa y cualitativa, de los tres ámbitos. En cuanto al efecto sectorial, éste expresa el impacto positivo o negativo del crecimiento de un sector específico, por arriba o por debajo de la tasa de crecimiento nacional. El efecto regional o competitivo recoge el dinamismo de un sector en una región, contrastándolo con ese mismo sector en el ámbito nacional(14).

Una vez obtenidos los resultados de los índices, se construyó una tabla para vincular la información con la tabla de datos de las áreas geoestadísticas municipales del marco geoestadístico 2013 del Instituto Nacional de Estadística, Geografía e Informática (INEGI). Usando software para análisis de sistemas de información geográfica (GIS por sus siglas en inglés) se elaboraron mapas para mostrar 
las diferencias regionales en cuanto a especialización espacial de la producción de leche.

\section{RESULTADOS}

Los resultados obtenidos en el periodo comprendido entre 2002 y 2013 muestran importantes cambios en cuanto a la posición de los DDR como productores de leche de bovino. De acuerdo al valor de la producción para 2002, las tres primeras posiciones correspondieron a los DDR Lagos de Moreno, Laguna Durango y Laguna Coahuila, en ese orden de importancia. Esta primacía se mantiene para 2013 aunque con cambios en su posición (Cuadro 1). Para 2002, sólo 18 de los 192 distritos pudieron considerarse como de alta producción de leche, y únicamente cuatro como de muy alta producción (DDR Chihuahua, Laguna Durango, Laguna Coahuila y Lagos de Moreno). Para el año 2013, 44 DDR se ubicaron como altos productores, seis de ellos como de muy alta producción. En ese mismo sentido, se dieron avances importantes en el centro del país; principalmente en Jalisco, Guanajuato, Querétaro e Hidalgo. La misma situación ocurrió en algunos DDR en el norte del país, principalmente en los estados de Chihuahua y Durango; y en el sur, Chiapas, Oaxaca y Veracruz (Figura 1). De estos últimos casos, resalta la entrada en escena de regiones con sistemas lecheros extensivos de doble propósito localizadas en los estados de Veracruz y Chiapas.

En cuanto a la especialización regional, el análisis del CL muestra que para 2002, 40 DDR del país se encontraban especializados en la producción

Cuadro 1. Principales distritos de desarrollo rural (DDR) productores de leche de bovino en México considerando el valor de la producción (VP)

\begin{tabular}{ccccc}
\hline Posición & DDR & $\begin{array}{c}\text { VP 2002 } \\
\text { (miles de pesos) }\end{array}$ & DDR & $\begin{array}{c}\text { VP 2013 } \\
\text { (miles de pesos) }\end{array}$ \\
\hline 1 & Lagos de Moreno & 3120530 & Laguna Coahuila & 7435896 \\
2 & Laguna Durango & 3099674 & Lagos de Moreno & 6340222 \\
3 & Laguna Coahuila & 3011539 & Laguna Durango & 5592601 \\
4 & Chihuahua & 2572113 & Delicias & 2511077 \\
5 & Celaya & 1821874 & Aguascalientes & 2106509 \\
\hline
\end{tabular}

Fuente: Elaboración propia con base en datos del SIAP (2014).

Figura 1. Regionalización por valor de la producción a) 2002 y b) 2013

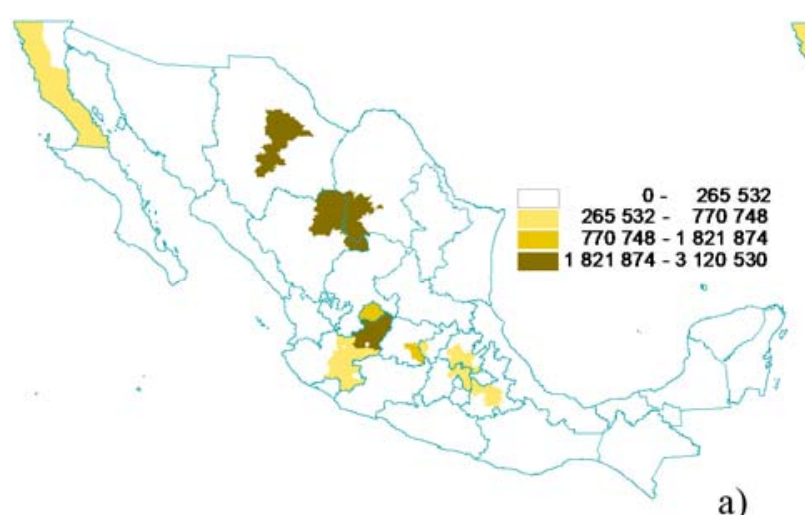

a)

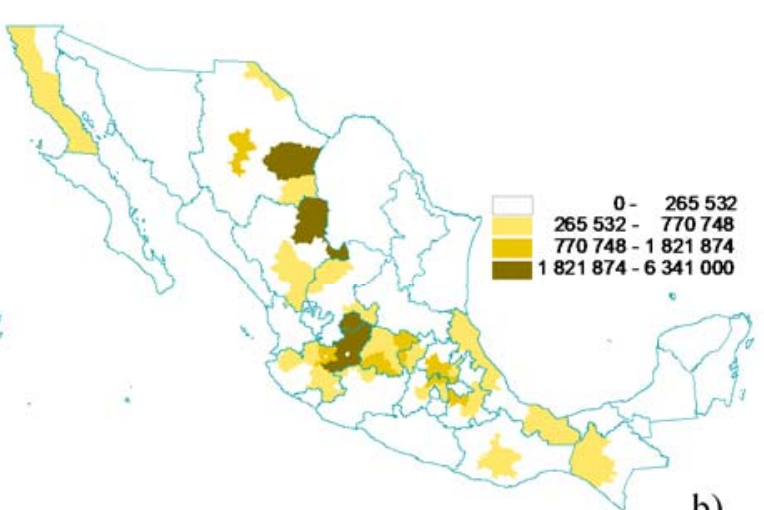

b) 
Cuadro 2. Grado de especialización de los distritos de desarrollo rural (DDR) de acuerdo al cociente de localización (CL) en el año 2002

\begin{tabular}{lccc}
\hline & No especializados & Especializados & Altamente especializados \\
\hline Rango (CL) & menor a 1 & $1-2.4$ & $2.4-4.7$ \\
No. de DDR & 151 & 33 & 7 \\
CL promedio & $0.38 \pm 0.25$ & $1.58 \pm 0.58$ & $3.95 \pm 0.45$ \\
\hline
\end{tabular}

Fuente: Elaboración propia con base en datos del SIAP (2014).

de leche. Siete de estos, clasificados como de alta especialización, en virtud de que su subsector lechero tuvo una importancia aproximadamente cuatro veces mayor $(\mathrm{CL}=3.95)$ que la que tuvo dicho subsector para el país en su conjunto. En adición, otros 33 DDR se clasificaron como especializados con un CL promedio de 1.58 (Cuadro 2). El mapa construido con esta información muestra claramente las regiones especializadas, dado que mediante el $\mathrm{CL}$ se sustraen del análisis aquéllas con valores menores a uno (no especializadas). Para 2002, se delimitaron 13 regiones especializadas (Figura 2), las cuales estuvieron constituidas de la siguiente manera: Región 1. En la península de Baja California conformada por los DDR Ensenada, Mulegé y Comondú. Región 2. Ubicada al norte del estado de sonora en el DDR Magdalena. Región 3. Al centro del estado de Chihuahua en el DDR Chihuahua. Región 4. Entre los estados de Durango y Coahuila conformada por los DDR Villa Ocampo, Laguna Durango y Laguna Coahuila. Región 5. Al centro y sur de Durango en el DDR Durango. Región 6. Conformada por la región centro y noreste del estado de Jalisco, en los DDR Colotlán, Lagos de Moreno, La Barca y Zapopan (zona Norte, Altos, Valle y Ciénega, en el estado de Jalisco). Considera también los DDR Tlaltenango (Zacatecas), Aguascalientes (Aguascalientes), Ojo Caliente (Zacatecas) y Sahuayo (Michoacán). Región 7. Integrada por los DDR Ciudad Valles y Ébano del estado de San Luis Potosí. Región 8. Conformada por los DDR San Juan del Río y Querétaro del estado de Querétaro, el DDR Celaya del estado de Guanajuato y el DDR Zitácuaro del estado de Michoacán. Región 9. Conformada por 11 DDR de cinco estados: Mixquiahuala y Pachuca del estado de Hidalgo; Zumpango, Toluca y Texcoco del Estado de México; Huamantla, Calpulalpan y Tlaxcala del estado de Tlaxcala; Coatepec del estado de Veracruz; Cholula y Libres del estado de Puebla. Región 10. DDR Lázaro Cárdenas en la costa del estado de Michoacán. Región 11. DDR Valles Centrales en el estado de Oaxaca. Región 12. DDR Pichucalco en la región norte del estado de Chiapas y Región 13. Integrada por los DDR Tonalá y Villa Flores de las regiones Istmo y La Frailesca del estado de Chiapas, respectivamente.

Para 2013 se aprecia un cambio sustancial en la configuración de la especialización respecto a 2002. El número de distritos considerados altamente especializados, prácticamente se duplicó, dado que pasó de 7 a 13. Además, se adicionan siete DDR en la categoría especializados para un total de 47 (Cuadro 3). También se puede observar que el límite superior de la clase de alta especialización tiene un valor mayor; es decir, se dieron casos de DDR mucho más especializados. Un ejemplo de este

Figura 2. Distritos de desarrollo rural (DDR) especializados (cociente de localización >1) en 2002

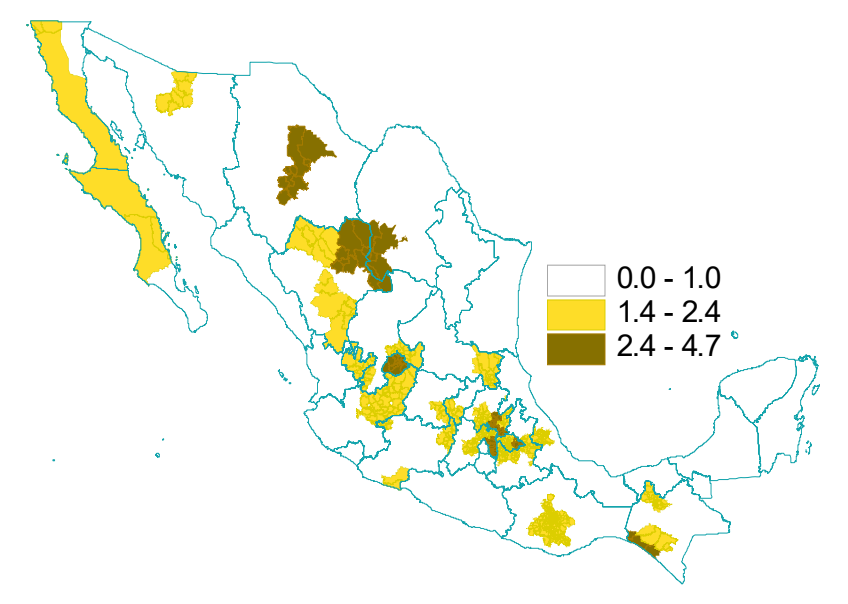

Fuente: Elaboración propia con base en datos del SIAP e INEGI (2014). 
Cuadro 3. Grado de especialización de los distritos de desarrollo rural (DDR) de acuerdo al cociente de localización (CL) en el año 2013

\begin{tabular}{lccc}
\hline & \multicolumn{3}{c}{ Grado de especialización } \\
\cline { 2 - 4 } & No especializados & Especializados & Altamente especializados \\
\hline Rango $(\mathrm{CL})$ & menor a 1 & $1-2.4$ & $2.4-6.2$ \\
No. de DDR & 144 & 34 & 13 \\
CL promedio & $0.37 \pm 0.29$ & $1.48 \pm 0.35$ & $3.5 \pm 1.08$ \\
\hline
\end{tabular}

Fuente: Elaboración propia con base en datos del SIAP (2014).

avance lo representa el caso Laguna Coahuila que pasó de un valor de $\mathrm{CL}$ de 4.5 a 6.2, lo que implica que para 2013 la importancia de la lechería en el sector agropecuario de esa región, fue aproximadamente seis veces mayor que la importancia que tuvo para la economía nacional.

En cuanto a otras modificaciones en regiones especializadas, en la península de Baja California, el DDR Comondú dejó de considerarse especializado, pero se agregó el DDR Los Cabos en Baja California Sur. El estado de Chihuahua se consolidó como una entidad especializada en la producción de leche, dado que al DDR Chihuahua, se sumaron los DDR Valle de Juárez, Casas Grandes, El Carmen, Madera, Cuauhtémoc, Delicias y Río Florido, con lo cual, prácticamente se forma un solo bloque con la región de la Laguna. De estos nuevos distritos, Juárez, Delicias y Río Florido se ubicaron en la categoría de alta especialización.

En la región 4, el DDR Villa Ocampo pierde su carácter de especializado; sin embargo, los dos DDR de la región Laguna permanecieron como altamente especializados. En la región 5 se adhirieron los DDR El Salto y Santiago Papasquiaro, en el estado de Durango. En la región 6 permanecen todos sus DDR como especializados; no obstante, la categoría de altamente especializados cambia: la pierde el DDR Aguascalientes y la adquiere el DDR Colotlán de la región norte de Jalisco y el DDR Sahuayo de Michoacán. Entre las regiones 6 y 8 aparecen como especializados los distritos de Dolores Hidalgo y San Luis de la Paz del estado de Guanajuato, con lo cual se conforma un corredor completo con la región 9 para conformar una macroregión. Por otra parte, el
DDR Celaya en el estado de Guanajuato de la región 8 y la región 7 dejan de ser especializados.

La región 9 crece con la adición del DDR Xochimilco (Ciudad de México) y Tulancingo (Hidalgo). No obstante, el DDR Libres (Puebla) y Coatepec (Veracruz) dejan de considerarse especializados. Los DDR Texcoco, Pachuca y Calpulalpan dejan de ser altamente especializados y pasan a esa categoría los DDR Cholula (Puebla) y Tulancingo (Hidalgo). Las regiones 10, 11 y 12 permanecen sin cambio en su estructura. Sin embargo, la región 13 pierde el DDR Villa Flores. Cabe señalar que surge una nueva región especializada en el norte del estado de Veracruz conformada por los DDR Martínez de la Torre y Tuxpan (Figura 3).

Figura 3. Distritos de desarrollo rural (DDR) especializados (cociente de localización >1) en 2013

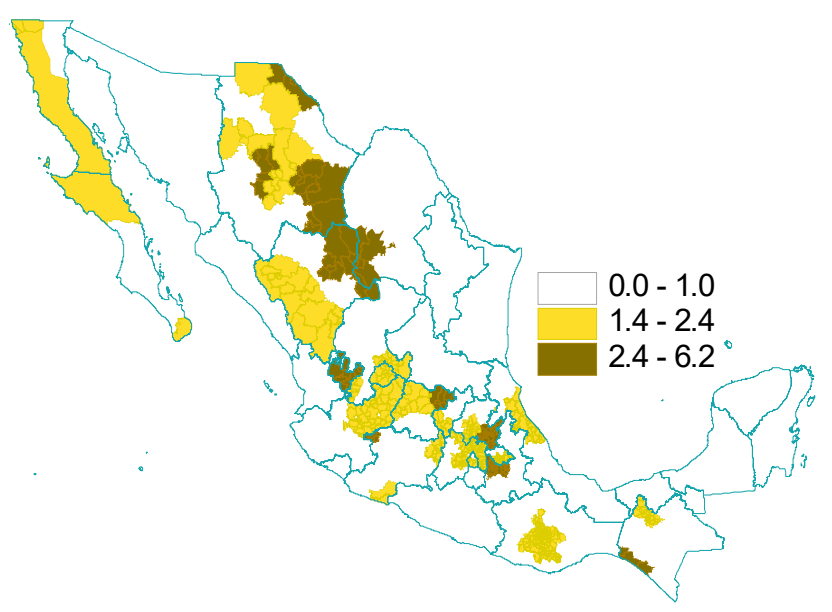

Fuente: Elaboración propia con base en datos del SIAP e INEGI (2014). 
Por otra parte, el análisis de cambio y participación mostró una serie de regiones con un comportamiento competitivo; es decir, DDR en donde el sector lechero tuvo un comportamiento más dinámico, donde una serie de especificidades regionales actuaron a favor de la actividad económica lechera. En total, 59 DDR distribuidos a lo largo del país (norte, centro y sur), evidenciaron esta cualidad (Figura 4a).

Algo destacable es que muchas de las regiones competitivas no corresponden con las especializadas, lo que podría indicar la presencia de zonas lecheras especializadas en declive, al menos durante el periodo analizado; y regiones no especializadas en ascenso. En total, sólo 16 DDR evidenciaron ambos comportamientos, una clara especialización y un efecto regional positivo (Figura 4b). Los DDR que presentan este comportamiento son: Los Cabos en Baja California Sur, Durango y El Salto en el estado de Durango, Laguna Coahuila en Coahuila, Ojo Caliente en Zacatecas, Lagos de Moreno y La Barca en Jalisco, Sahuayo en Michoacán, San Juan del Río en Querétaro, Mixquiahuala en Hidalgo, Jilotepec y Toluca en el Estado de México, Cholula en Puebla, Martínez de la Torre en Veracruz y Tonalá en Chiapas.

\section{DISCUSIÓN}

Prácticamente en todos los estados de la República Mexicana (con excepción de Chihuahua,
Baja California, Aguascalientes, Guanajuato, Tlaxcala, Yucatán y Quintana Roo) hay un DDR en el cual la actividad lechera muestra un crecimiento superior al manifestado por la economía nacional y por el subsector en general (Figura 4a). Sin embargo, sólo en 16 de los 47 DDR especializados se expresó esa misma tendencia (Figura 4b). En otras palabras, en 31 regiones especializadas se percibió un comportamiento menos robusto de la actividad. Tal situación podría hacer evidente la saturación en el uso de recursos de las zonas tradicionalmente lecheras, lo que conllevaría a la búsqueda de regiones de producción alternativas, con la consecuente expansión de la producción lechera, o por lo menos la conversión de la agricultura hacia la producción de cultivos forrajeros. El aumento de las regiones especializadas y el comportamiento dinámico en buena parte del país, podrían tomarse como evidencias del avance de lo que se ha denominado modelo Holstein de lechería industrial. Es de suponerse que en las regiones donde se manifestó un avance de la actividad lechera, se generó también una ganaderización de la agricultura, con la consecuente reconversión de los cultivos hacia la producción de forrajes, como ha sucedido en la región de La Laguna ${ }^{(15)}$.

Al igual que en las zonas norte y noroeste del país, en el centro y centro-occidente se muestra un avance tanto en la especialización de las regiones, como en el establecimiento de condiciones favorables para el desarrollo de la lechería. En esta

Figura 4. a) Regiones lecheras competitivas y b) especializadas y competitivas

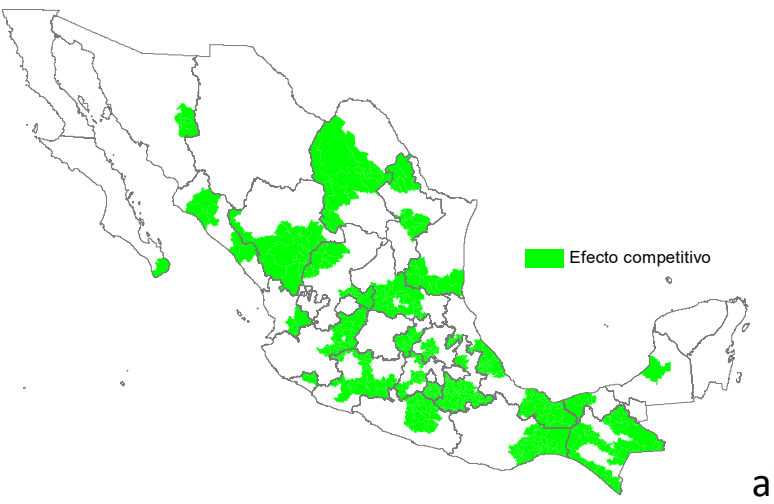

a)

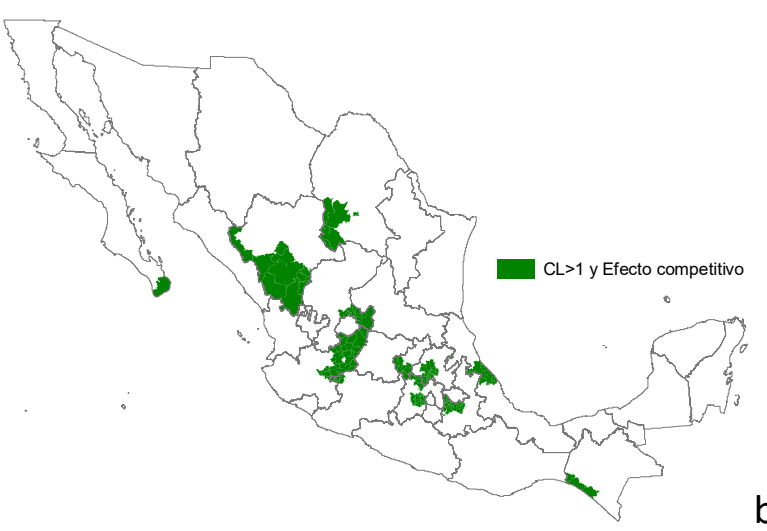

b)

Fuente: Elaboración propia con base en datos del SIAP e INEGI (2014). 
región coexiste la lechería familiar (v.g. Jalisco) y la lechería altamente tecnificada (v.g. Querétaro), ambas ligadas estrechamente a la agroindustria de mediana y gran escala. Tanto la industria nacional, como la transnacional operan bajo la misma lógica de integración con el sector primario. Incluso la mediana y pequeña industria de transformación se relacionan con los productores bajo una lógica de desequilibrio de poder, que les permite establecer el precio de manera unilateral ${ }^{(16)}$.

Las regiones tropicales con lechería de doble propósito manifiestan también una dinámica positiva. La zona sur de Oaxaca, Chiapas prácticamente en su totalidad (a excepción de la zona montañosa en la Sierra Madre de Chiapas), la región sur de Veracruz y la región Chontalpa de Tabasco, muestran la existencia de condiciones favorables que promovieron un mayor dinamismo de la actividad, a pesar de que únicamente la región de La Frailesca en Chiapas y Martínez de la Torre en Veracruz manifestaron un grado de especialización en la producción de leche.

En términos absolutos, se hace evidente el avance de la lechería en el país, lo que es comprensible si se considera que la evolución de la producción de leche está íntimamente ligada al crecimiento de la población. Esta tendencia continúa aún a pesar de los volúmenes de importación de leche en polvo y productos derivados con alta capacidad de sustituir a la leche líquida. Aumento que para el periodo de análisis fue de $14.6 \%$, de acuerdo con datos del Banco Mundial(17). Tanto la lechería intensiva del norte del país como la semiintensiva familiar y la de doble propósito muestran avances importantes durante los últimos doce años.

Destaca de manera particular el desarrollo de los niveles de producción lechera de las zonas tropicales. Este hecho compagina con el avance registrado durante las dos décadas anteriores, desarrollo explicado en parte por la crisis de los sistemas intensivos durante ese periodo, y por la intensificación productiva derivada de la introducción de pastos mejorados a mediados de la década de los $80 \mathrm{~s}^{(5)}$. De esta manera, se puede afirmar que durante la década en estudio se continúa con la tendencia de crecimiento acelerado de la lechería de doble propósito en regiones tropicales.

A pesar de los contratiempos de los sistemas altamente productivos del norte y centro del país, la "lecherización" de las economías agropecuarias de los estados muestra un avance vigoroso. Pudiera pensarse como una situación paradójica, el hecho de que en regiones con altos grados de especialización y condiciones de aparente competitividad (v.g. La Laguna, Los Altos de Jalisco, Puebla, Zacatecas, entre otras), se manifiesten desde hace varios años, problemas de bajos precios, abandono de la

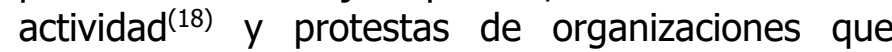
exigen la intervención del Estado para garantizar el precio de su producto. Sin embargo, lejos de ser un contrasentido, los conflictos emergentes son parte de la presión competitiva causada por la importación de leche en polvo. Cuando la industria introduce este insumo a sus procesos, el efecto es el de una sobreoferta de leche en el mercado local y regional, con la consecuente disminución del precio pagado al productor. Dado que la leche importada proviene de sistemas con una productividad mayor proporcionada por la intensidad de uso de capital, con altos niveles de subsidio y transferencias indirectas por parte del Estado (v.g. Estados Unidos), la producción nacional tiene pocas posibilidades de competir y se ve obligada a disminuir su precio como una estrategia para permanecer en la actividad.

Son recurrentes las quejas por parte de productores individuales y organizaciones de productores lecheros, sobre la imposibilidad para cubrir los costos de producción dados los bajos precios que reciben por parte de la industria. En el mismo sentido, es frecuente que sobrevengan protestas ante las dependencias relacionadas con el sector, en las que de manera reiterada se exige la disminución o la desaparición de la importación de leche en polvo, cuyo precio internacional actúa como precio de indiferencia que la industria toma para definir el precio que se paga a productores. No obstante, la política pública privilegia el abastecimiento de los programas de apoyo social, bajo el argumento de la seguridad alimentaria. Por medio de la empresa de participación estatal LICONSA, se importa una gran cantidad de leche en 
polvo (principalmente de Estados Unidos) que, una vez en el país, es reconstituida para abastecer con leche barata a los habitantes urbanos beneficiarios de los programas ${ }^{(18)}$. En total, México importa alrededor del $30 \%$ del consumo nacional de leche, lo que implica que diariamente, en cada entidad con actividad lechera, se dejen de comprar millones de litros a los productores nacionales ${ }^{(19)}$. La situación descrita provoca varias consecuencias que van desde el sacrificio de ganado hasta la salida definitiva de la actividad económica. Este escenario no es privativo de los sistemas familiares 0 extensivos basados en razas especializadas, en los sistemas de doble propósito vinculados con agroindustrias transnacionales como Nestlé, o con la agroindustria local, la situación es similar. Estos conflictos solo demuestran la capacidad de la agroindustria para imponer las condiciones de mercado (precio y calidad) y su influencia sobre las políticas gubernamentales; es decir, el avance del modelo Holstein en el país.

Al igual que en otros países, en México existe una tendencia hacia la concentración de la producción de leche que está relacionada de manera directa con el avance del modelo Holstein; lo cual se deriva, normalmente, de las exigencias de calidad, en donde los pequeños productores han quedado, generalmente, segregados y desplazados de los mercados, dada su limitada capacidad para producir los grandes volúmenes requeridos por las empresas industrializadoras del lácteo ${ }^{(6)}$. Al mismo tiempo que la actividad va incrementando, su predominio espacial como actividad económica, se dan procesos de concentración de la producción generados por la salida constante de productores que se ven obligados a cambiar de actividad por no poder soportar los bajos precios, es decir, por no ser competitivos. Esto es cierto para todas las regiones donde la lechería está vinculada con la agroindustria, pero es especialmente relevante en aquéllas que tienen cierto grado de especialización. Lo anterior incluye a los tres sistemas productivos de leche de bovino (intensivo, familiar y doble propósito). Se podría pensar que en el caso del sistema doble propósito la situación sería diferente; sin embargo, el desequilibrio de poder con empresas como Nestlé y la difusión del modelo tecnológico Holstein (inseminación artificial, ordeña mecanizada, uso de concentrados como complemento del pastoreo, semiestabulación, etc.) generan las mismas situaciones que se presentan en los otros sistemas de producción de leche.

Es de esperarse que en las regiones con mayor producción lechera y más especializadas en esta actividad, los pequeños y medianos productores primarios tengan fuertes presiones para ser competitivos; es decir, para cumplir con las condiciones de las plantas pasteurizadoras y procesadoras $^{(20)}$. En esas situaciones la distribución directa del producto y la transformación de la leche en derivados artesanales (v.g. quesos, yogurt, etc.), pueden constituirse como estrategias de resistencia y de sobrevivencia del productor, si se consigue el acceso a mercados que valoren las características de estos productos derivados de la leche.

\section{CONCLUSIONES E IMPLICACIONES}

Los tres indicadores analizados en este estudio muestran que entre 2002 y 2013 se dio una reconfiguración espacial de la lechería. En general se puede afirmar que en el país se han incrementado las regiones especializadas, lo que implica que, en el ámbito nacional, la producción de leche de bovino ha ganado preponderancia con respecto a otras actividades del sector agropecuario. En la última década, el modelo Holstein ha tenido un avance significativo y las regiones tradicionalmente consideradas como de especialización lechera han expandido ampliamente sus fronteras. En ese sentido, zonas como La laguna han mantenido su estatus alto, al mismo tiempo entidades como Chihuahua y Durango se han consolidado como regiones de alta especialización. Una situación similar ocurre en el centro del país en donde se conforma una macro región especializada que fusiona prácticamente todo el altiplano central de México, desde Zacatecas hasta Tlaxcala. En ese mismo sentido, regiones como el norte del estado de Veracruz y Chiapas han orientado aún más su estructura agropecuaria hacia la producción de leche de bovino. Por lo expuesto, se presupone un fuerte avance del modelo Holstein en toda la lechería del país. Las consecuencias se hacen ya evidentes, el 
aumento en el desequilibrio de poder en la relación entre el sector primario y la transformación, desaparición o pauperización de pequeños productores y dependencia tecnológica. En ese mismo sentido sería relevante, para futuras investigaciones, analizar las consecuencias socioeconómicas y ambientales de la reconversión del uso del suelo de agrícola a ganadero.

\section{LITERATURA CITADA}

1 Fischler C. El (h) omnívoro: el gusto, la cocina y el cuerpo. Barcelona: Editorial Anagrama; 1995.

2. Rubio B. El dominio del hambre: Crisis de hegemonía y alimentos. 1er ed. México, Editorial Juan Pablos; 2014.

3. Martínez E, Álvarez A, García LA, Del Valle MC. Dinámica del sistema lechero mexicano en el marco regional y global. México: Plaza y Valdés; 1999.

4. Vatin F. L' industrie du lait. Essai d'histoire économique. L'Harmattan, París; 1991.

5. Del Valle MC. La innovación tecnológica en el sistema lácteo mexicano y su entorno mundial. Universidad Nacional Autónoma de México, Instituto de Investigaciones Económicas, Miguel Ángel Porrúa; 2000.

6. Pomeón $T$, Boucher F, Cervantes F, Fournier S. Las dinámicas colectivas en dos cuencas lecheras mexicanas: Tlaxco, Tlaxcala y Tizayuca. Agroalimentaria 2006;(22):49-64.

7. García LA. Estrategias de las agroindustrias lecheras latinoamericanas. Estudio comparativo ante el proceso de globalización económica. Rev Mex Agroneg 2001;(9):263-273.

8. Del Valle MC. Sistema de innovación y transformaciones socioeconómicas en la agroindustria de los quesos en México [resumen]. Congreso de la de la Asociación Latinoamericana de Sociología Rural (ALASRU). Quito, Ecuador. 2007:24.
9. Arias $\mathrm{JA}$, Fortich $\mathrm{FJ}$. El panorama teórico de la economía regional y los modelos de análisis territorial. Rev Finanzas y Política Económ 2010;2(2):9-26.

10. Boisier $S$. Técnicas de análisis regional con información limitada. Cuaderno ILPES, Serie II, N²7, Santiago de Chile; 1980.

11. Hair FJ, Black WC, Babin BJ, Anderson RE. Multivariate data analysis. 7a ed. USA: Prentice Hall; 2010.

12. Dunn ES. A statistical and analytical technique for regional analysis. Pap Proc Reg Sci Ass 1960;(6):97-112.

13. Salazar $\mathrm{H}$. Críticas y correcciones a la técnica de análisis de cambio y participación. Demografía y economía 1983;17(1):21-37.

14. Mayor M, López AJ. El análisis shift-share espacial: nuevos desarrollos. VIII Encuentro de economía aplicada-España, Facultad de Economía y Empresas, Universidad de Murcia, España; 2005.

15. Ríos FJM, Torres MM, Ramírez GM, Caamal CI, Jerónimo AF, Márquez DL. Productividad y ganaderización del sector agrícola en la laguna, México, de 1990 a 2005. Rev Chapingo, Serie Zonas Áridas 2009;(8):141-147.

16. Camacho $\mathrm{JH}$, Noriega $\mathrm{F}$. Análisis del sistema productivo leche de bovino en el municipio de Tecamachalco, Puebla. Bernal H, Ramírez B, coord. Estudios para el desarrollo rural de Puebla. Puebla, México: Colegio de Postgraduados y Altres Costa-Amic Editores; 2014.

17. Banco Mundial (BM). Indicadores de desarrollo para México. http://databank.bancomundial.org/data/reports.aspx?source=2\&c ountry=MEX\&series=\&period = 2014. Consultado 25 Sep, 2015.

18. Cervantes $F$, Cesín A, Pérez $S$. El abandono de la ganadería lechera y reconversión productiva en Chipilo, Puebla. Téc Pecu Méx 2007;45(2):195-208.

19. Cervantes F, Camacho J, Cesín A. El sistema lácteo mexicano después del TLCAN. Caballotti $\mathrm{B}$, et al coord. La ganadería mexicana a 20 años del Tratado de Libre Comercio de América del Norte. Universidad Autónoma Chapingo. 2014.

20. Roman JA. (2015, 20 de abril). Productores de leche, en crisis inédita; apelan a la voluntad política del gobierno. La Jornada. en http://www.jornada.unam.mx/2015/04/20/politica/014n1pol. Consultado 25 Sep, 2015. 